\title{
Long Range Scattering for Nonlinear Schrödinger Equations in One Space Dimension
}

\section{Tohru Ozawa}

Laboratoire de Physique Théorique et Hautes Energies, ${ }^{\star \star}$ Université de Paris XI, Bâtiment 211, F-91405 Orsay, France

Received November 13, 1990

Abstract. We consider the scattering problem for the nonlinear Schrödinger equation in $1+1$ dimensions:

$$
\mathrm{i} \partial_{t} u+(1 / 2) \partial^{2} u=\lambda|u|^{2} u+\mu|u|^{p-1} u, \quad(t, x) \in \mathbb{R} \times \mathbb{R},
$$

where $\partial=\partial / \partial x, \lambda \in \mathbb{R} \backslash\{0\}, \mu \in \mathbb{R}, p>3$. We show that modified wave operators for (*) exist on a dense set of a neighborhood of zero in the Lebesgue space $L^{2}(\mathbb{R})$ or in the Sobolev space $H^{1}(\mathbb{R})$. The modified wave operators are introduced in order to control the long range nonlinearity $\lambda|u|^{2} u$.

\section{Introduction}

In this paper we consider the asymptotic behavior in time of solutions to the Schrödinger equations with power nonlinearities:

$$
i \partial_{t} u+(1 / 2) \partial^{2} u=f(u), \quad(t, x) \in \mathbb{R} \times \mathbb{R},
$$

where $u$ is a complex valued function on $\mathbb{R} \times \mathbb{R}, \partial_{t}=\partial / \partial t, \partial=\partial / \partial x$, and $f$ is a complex valued function on $\mathbb{C}$. A typical form of $f(u)$ is the sum of two powers

$$
f(u)=\lambda|u|^{q-1} u+\mu|u|^{p-1} u
$$

with $p \geqq q \geqq 1, \lambda, \mu \in \mathbb{R}$.

There is a large literature on the equations of the form (1.1) from both mathematical and physical point of view, see [1-4, 7-17, 19-26, 28-30]. Let $H^{m, s}$ be the weighted Sobolev space defined by

$$
H^{m, s}=\left\{\psi \in \mathscr{S}^{\prime} ;\|\psi\|_{m, s}=\left\|\left(1+|x|^{2}\right)^{s / 2}\left(1-\partial^{2}\right)^{m / 2} \psi\right\|_{2}<\infty\right\}, \quad m, s \in \mathbb{R},
$$

\footnotetext{
* On leave of absence from Research Institute for Mathematical Sciences, Kyoto University, Kyoto 606, Japan

$\star \star$ Laboratoire associé au Centre National de la Recherche Scientifique
} 
where $\|\cdot\|_{p}$ denotes the norm in $L^{p}=L^{p}(\mathbb{R})$. We denote by $U(t)$ the free propagator $\exp \left(i(t / 2) \partial^{2}\right)$. Concerning the Cauchy problem for $(1.1)$, the following results are well known.

(1) If $5>p \geqq q \geqq 1$, then for any $\phi \in L^{2}$ (1.1) has a unique solution $u \in C\left(\mathbb{R} ; L^{2}\right) \cap L_{\mathrm{loc}}^{4(p+1) /(p-1)}\left(\mathbb{R} ; L^{p+1}\right)$ with $\partial_{t} u \in L_{\mathrm{loc}}^{4(p+1) /(p-1)}\left(\mathbb{R} ; H^{-2,0}\right)$ and $u(0)=\phi$ $([2,16,24])$. Moreover, if $\phi \in H^{1,0}$, then $u \in C\left(\mathbb{R} ; H^{1,0}\right) \cap C^{1}\left(\mathbb{R} ; H^{-1,0}\right)([2,16])$.

(2) Assume one of the following three conditions: (a) $5>p \geqq q \geqq 1$. (b) $p \geqq 5>q \geqq 1$ with $\mu \geqq 0$. (c) $p \geqq q \geqq 1$ with $\lambda, \mu \geqq 0$. Then for any $\phi \in H^{1,0}(1.1)$ has a unique solution $u \in C\left(\mathbb{R} ; H^{1,0}\right) \cap C^{1}\left(\mathbb{R} ; H^{-1,0}\right)$ with $u(0)=\phi([2,3,8,15,16])$.

(3) If $p \geqq q \geqq 5$ with $\lambda, \mu<0$, then (1.1) has blow-up solutions $([2,10,26])$.

Concerning the asymptotic behavior in time of solutions and the scattering theory, the following results are well known.

(I) If $p \geqq q>(3+\sqrt{17}) / 2$, then there exists $\varepsilon_{0}>0$ with the following properties: For any $\phi_{+} \in H^{1,0}$ with $\left\|\phi_{+}\right\|_{1,0}+\left\|\phi_{+}\right\|_{(p+1) / p}<\varepsilon_{0}$ (1.1) has a unique solution $u \in C\left(\mathbb{R} ; H^{1,0}\right)$ such that

$$
\left\|U(-t) u(t)-\phi_{+}\right\|_{1,0} \rightarrow 0 \text { as } t \rightarrow+\infty .
$$

For any $\phi_{-} \in H^{1,0}$ with $\left\|\phi_{-}\right\|_{1,0}+\left\|\phi_{-}\right\|_{(p+1) / p}<\varepsilon_{0}$ (1.1) has a unique solution $u \in C\left(\mathbb{R} ; H^{1,0}\right)$ such that

$$
\left\|U(-t) u(t)-\phi_{-}\right\|_{1,0} \rightarrow 0 \text { as } t \rightarrow-\infty .
$$

For any $\phi \in H^{1,0}$ with $\|\phi\|_{1,0}+\|\phi\|_{(p+1) / p}<\varepsilon_{0}$ there exist unique $\phi_{ \pm} \in H^{1,0}$ satisfying $(1.3)_{ \pm}$, where $u$ is a unique solution of $(1.1)$ with $u(0)=\phi([9,19])$.

(II) If $p \geqq q>3$ with $\lambda, \mu>0$, then for any $\phi \in H^{1,0} \cap H^{0,1}$ there exist unique $\phi_{ \pm} \in L^{2}$ such that

$$
\left\|U(-t) u(t)-\phi_{ \pm}\right\|_{2} \rightarrow 0 \text { as } t \rightarrow \pm \infty
$$

where $u$ is a unique solution of (1.1) with $u(0)=\phi([25])$. If $5 \geqq p \geqq q>3$ with $\lambda, \mu>0$, then for any $\phi \in H^{0,1}$ there exist unique $\phi_{ \pm} \in L^{2}$ satisfying (1.4) $)_{ \pm}([12])$.

(III) If $3 \geqq q \geqq 1$ with $\lambda \neq 0, \mu>0$, then for any $\phi \in H^{1,0} \backslash\{0\}$ there do not exist any $\phi_{ \pm} \in L^{2}$ satisfying $(1.4)_{ \pm}$. If $3 \geqq q \geqq 1,5>p \geqq q$ with $\lambda \neq 0$, then for any $\phi \in L^{2} \backslash\{0\}$ there do not exist any $\phi_{ \pm} \in L^{2}$ satisfying $(1.4)_{ \pm}([7,14,23])$.

As we see above, a critical number of the $L^{2}$-scattering theory is $q=3$. In the case $p \geqq q>3$ with $\lambda, \mu>0$, any solution $u$ of $(1.1)$ with $u(0) \in H^{1,0} \cap H^{0,1}$ behaves like free solutions $U(t) \phi_{ \pm}$as $t \rightarrow \pm \infty$. This is because the dispersive effect is stronger than the nonlinear effect as $t \rightarrow \pm \infty$ when $q>3$. In the case $3 \geqq q \geqq 1$ the nonlinear effect is dominant and any nontrivial solution does not behave like free solutions. If we regard the nonlinear factor $\lambda|u|^{q-1}+\mu|u|^{p-1}$ as a potential, the $L^{\infty}$-norm of the potential is estimated as $O\left(|t|^{-(q-1) / 2}\right)$ as $t \rightarrow \pm \infty$ since $\|u(t)\|_{\infty}=O\left(|t|^{-1 / 2}\right)$ when $\lambda, \mu>0$. We then associate the borderline $q=3$ with the decay rate $O\left(|t|^{-1}\right)$ of the potential. The same analogy works in the higher dimensional cases or in potential scattering. In $n$-dimensional cases the breakdown of scattering for the nonlinearity $f(u)=|u|^{q-1} u$ occurs if and only if $q \leqq 1+2 / n$. The borderline $q=1+2 / n$ corresponds to the decay rate $O\left(|t|^{-1}\right)$ of the potential $|u|^{2 / n}$, since $\|u(t)\|_{\infty}=O\left(|t|^{-n / 2}\right)$. For the potential $V(x)=\lambda|x|^{-\gamma}$, $x \in \mathbb{R}^{n}, n \geqq 3$, the existence and completeness of the usual wave operators s-limexp $\left(i t\left(H_{0}+V\right)\right) \exp \left(-i t H_{0}\right)$ break down if and only if $\gamma \leqq 1$, where $H_{0}=$ $t \rightarrow \pm \infty$

$-(1 / 2) \Delta$ and $\Delta$ denotes the Laplacian in $\mathbb{R}^{n}[18]$. The corresponding decay condition of the potential should be replaced by the estimate $\left\||x|^{-\gamma} \exp \left(-i t H_{0}\right) \phi\right\|_{2}=O\left(|t|^{-\gamma}\right)$ 
for any $\phi \in L^{2}$ with the Fourier transform $\hat{\phi} \in C_{0}^{\infty}\left(\mathbb{R}^{n}\right)$. The borderline $\gamma=1$ then corresponds to the same decay rate $O\left(|t|^{-1}\right)$ as before. It is customary that potentials of the decay rate $O\left(|x|^{-\gamma}\right)$ as $|x| \rightarrow \infty$ with $\gamma \leqq 1$ are called long range potentials. In the long range case we know that the comparison dynamics $U(t) \phi_{ \pm}$ should be replaced by a modified free evolution in order to take the long range interaction into account.

Our purpose in this paper is to find a comparison dynamics for solutions of (1.1) in the critical case $q=3$. In order to state the main results we make the following hypotheses and definitions. In the following we assume that the nonlinear term $f(u)$ takes the form

$$
f(u)=\lambda|u|^{2} u+\mu|u|^{p-1} u
$$

with $\lambda \in \mathbb{R} \backslash\{0\}$ and $\mu \in \mathbb{R}$. For $\phi_{ \pm} \in L^{2}$ we define the phase functions $S^{ \pm}(t, x)$ and $S_{0}^{ \pm}(t, x)$ by

$$
\begin{aligned}
& S^{ \pm}(t, x)=\mp \lambda \log |t|\left|\hat{\phi}_{ \pm}\left(t^{-1} x\right)\right|^{2} \pm(2 \mu /(p-3))|t|^{-(p-3) / 2}\left|\hat{\phi}_{ \pm}\left(t^{-1} x\right)\right|^{p-1} . \\
& S_{0}^{ \pm}(t, x)=\mp \lambda \log |t|\left|\hat{\phi}_{ \pm}\left(t^{-1} x\right)\right|^{2},
\end{aligned}
$$

respectively, where ${ }^{\wedge}$ denotes the Fourier transform defined by

$$
\hat{\psi}(\xi)=(2 \pi)^{-1 / 2} \int \exp (-i x \xi) \psi(x) d x .
$$

For any function $(t, x) \mapsto w(t, x)$ we denote by $w(t)$ the function $x \mapsto w(t, x)$.

Theorem 1. Let $3<p<5$. Then there exists $\varepsilon_{1}>0$ with the following properties:

(1) For any $\phi_{+} \in H^{0,2}$ with $\left\|\hat{\phi}_{+}\right\|_{\infty}<\varepsilon_{1}$ (1.1) has a unique solution $u \in C\left(\mathbb{R} ; L^{2}\right) \cap L_{\mathbf{l o c}}^{4}\left(\mathbb{R} ; L^{\infty}\right)$ such that for any $\alpha$ with $1 / 2<\alpha<1$,

$$
\begin{gathered}
\left\|u(t)-\exp \left(i S^{+}(t)\right) U(t) \phi_{+}\right\|_{2}=O\left(t^{-\alpha}\right) \\
\left(\int_{t}^{+\infty}\left\|u(\tau)-\exp \left(i S^{+}(\tau)\right) U(\tau) \phi_{+}\right\|_{\infty}^{4} d \tau\right)^{1 / 4}=O\left(t^{-\alpha}\right) \text { as } t \rightarrow+\infty .
\end{gathered}
$$

(2) For any $\phi_{-} \in H^{0,2}$ with $\left\|\hat{\phi}_{-}\right\|_{\infty}<\varepsilon_{1}$ (1.1) has a unique solution $u \in C\left(\mathbb{R} ; L^{2}\right) \cap L_{\text {loc }}^{4}\left(\mathbb{R} ; L^{\infty}\right)$ such that for any $\alpha$ with $1 / 2<\alpha<1$,

$$
\begin{gathered}
\left\|u(t)-\exp \left(i S^{-}(t)\right) U(t) \phi_{-}\right\|_{2}=O\left(|t|^{-\alpha}\right) \\
\left(\int_{-\infty}^{t}\left\|u(\tau)-\exp \left(i S^{-}(\tau)\right) U(\tau) \phi_{-}\right\|_{\infty}^{4} d \tau\right)^{1 / 4}=O\left(|t|^{-\alpha}\right) \text { as } t \rightarrow-\infty .
\end{gathered}
$$

Corollary 1. Let $\phi_{ \pm}$and $u$ be as in Theorem 1. Then:

(1) For any $\alpha$ with $1 / 2<\alpha<1$,

$$
\left\|u(t)-\exp \left(i S_{0}^{ \pm}(t)\right) U(t) \phi_{ \pm}\right\|_{2}=O\left(|t|^{-\tilde{\alpha}}\right) \quad \text { as } t \rightarrow \pm \infty
$$

where $\tilde{\alpha}=\min (\alpha,(p-3) / 2)$ if $\mu \neq 0$ and $\tilde{\alpha}=\alpha$ if $\mu=0$.

(2) For any $\alpha$ with $1 / 2<\alpha<1$,

$$
\begin{aligned}
\left\||u(t)|^{2}-\left|U(t) \phi_{ \pm}\right|^{2}\right\|_{1} & =O\left(|t|^{-\alpha}\right), \\
\left\||u(t)|-\left|U(t) \phi_{ \pm}\right|\right\|_{2} & =O\left(|t|^{-\alpha / 2}\right) \text { as } t \rightarrow \pm \infty . \\
\left(\int_{t}^{ \pm \infty}\|u(\tau)\|_{\infty}^{4} d \tau\right)^{1 / 4} & =O\left(|t|^{-1 / 4}\right) \text { as } t \rightarrow \pm \infty .
\end{aligned}
$$


Theorem 2. Let $p>3$. Suppose $\mu \geqq 0$ when $p \geqq 5$. Then there exists $\varepsilon_{2}>0$ with the following properties:

(1) For any $\phi_{+} \in H^{0,3} \cap H^{1,2}$ with $\left\|\hat{\phi}_{+}\right\|_{\infty}<\varepsilon_{2} \quad\left(\left\|\hat{\phi}_{+}\right\|_{\infty}+\widehat{\partial \phi}_{+} \|_{\infty}<\varepsilon_{2}\right.$ if $p \geqq 5)(1.1)$ has a unique solution $u \in C\left(\mathbb{R} ; H^{1,0}\right) \cap L_{\text {loc }}^{4}\left(\mathbb{R} ; W^{1, \infty}\right)$ such that for any $\alpha$ with $1 / 2<\alpha<1$,

$$
\begin{array}{r}
\left\|u(t)-\exp \left(i S^{+}(t)\right) U(t) \phi_{+}\right\|_{1,0}=O\left(t^{-\alpha}\right), \\
\left(\int_{t}^{+\infty}\left\|u(\tau)-\exp \left(i S^{+}(\tau)\right) U(\tau) \phi_{+}\right\|_{W^{1, \infty}}^{4} d \tau\right)^{1 / 4}=O\left(t^{-\alpha}\right)
\end{array}
$$

as $t \rightarrow+\infty$, where $W^{1, \infty}=\left\{\psi \in L^{\infty} ; \partial \psi \in L^{\infty}\right\},\|\psi\|_{W^{1, \infty}}=\|\psi\|_{\infty}+\|\partial \psi\|_{\infty}$.

(2) For any $\phi_{-} \in H^{0,3} \cap H^{1,2}$ with $\left\|\hat{\phi}_{-}\right\|_{\infty}<\varepsilon_{2}\left(\left\|\hat{\phi}_{-}\right\|_{\infty}+\|\widehat{\partial \phi}-\|_{\infty}<\varepsilon_{2}\right.$ if $\left.p \geqq 5\right)$ (1.1) has a unique solution $u \in C\left(\mathbb{R} ; H^{1,0}\right) \cap L_{\text {loc }}^{4}\left(\mathbb{R} ; W^{1, \infty}\right)$ such that for any $\alpha$ with $1 / 2<\alpha<1$,

$$
\begin{gathered}
\left\|u(t)-\exp \left(i S^{-}(t)\right) U(t) \phi_{-}\right\|_{1,0}=O\left(|t|^{-\alpha}\right) \\
\left(\int_{-\infty}^{t}\left\|u(\tau)-\exp \left(i S^{+}(\tau)\right) U(\tau) \phi_{-}\right\|_{W^{1, \infty}}^{4} d \tau\right)^{1 / 4}=O\left(|t|^{-\alpha}\right) \text { as } t \rightarrow-\infty .
\end{gathered}
$$

Corollary 2. Let $\phi_{ \pm}$and $u$ be as in Theorem 2. Then:

(1) For any $\alpha$ with $1 / 2<\alpha<1$,

$$
\begin{gathered}
\left\|u(t)-\exp \left(i S_{0}^{ \pm}(t)\right) U(t) \phi_{ \pm}\right\|_{1,0}=O\left(|t|^{-\tilde{\alpha}}\right) \quad \text { as } t \rightarrow \pm \infty . \\
\|u(t)\|_{\infty}=O\left(|t|^{-1 / 2}\right) \text { as } t \rightarrow \pm \infty
\end{gathered}
$$

Remark. (1) By the inequalities

$$
\begin{aligned}
\|\hat{\psi}\|_{\infty} & \leqq(2 \pi)^{-1 / 2}\|\psi\|_{1} \\
& \leqq(2 \pi)^{-1 / 2} \inf _{\rho>0}\left\|\left(\rho^{2}+x^{2}\right)^{-1 / 2}\right\|_{2}\left(\rho^{2}\|\psi\|_{2}^{2}+\|x \psi\|_{2}^{2}\right)^{1 / 2} \\
& =\|\psi\|_{2}^{1 / 2}\|x \psi\|_{2}^{1 / 2} \leqq\|\psi\|_{0,1},
\end{aligned}
$$

we see that $\left\|\hat{\phi}_{ \pm}\right\|_{\infty}<\varepsilon$ follow from either $\left\|\phi_{ \pm}\right\|_{1}<\varepsilon$ or $\left\|\phi_{ \pm}\right\|_{0,1}<\varepsilon$ and that $\left\|\hat{\partial \phi_{ \pm}}\right\|_{\infty}<\varepsilon$ follows from either $\left\|\partial \phi_{ \pm}\right\|_{1}<\varepsilon$ or $\left\|\partial \phi_{ \pm}\right\|_{0,1}<\varepsilon$.

(2) In the case where $\hat{\phi}_{ \pm}$have compact support, the assumptions $\left\|\hat{\phi}_{ \pm}\right\|_{\infty}<\varepsilon$ and $\left\|\hat{\partial \phi_{ \pm}}\right\|_{\infty}<\varepsilon$ may be replaced by the condition $\left|\hat{\phi}_{ \pm}(0)\right|<\delta$ for some $\delta>0$. This follows from a slight modification of the proof given in the next section. Note that $\hat{\phi}_{ \pm}$are continuous on $\mathbb{R}$.

Theorems 1 and 2 show that in the long range case (1.1) has solutions which behave like $\exp \left(i S^{ \pm}(t)\right) U(t) \phi_{ \pm}$as $t \rightarrow \pm \infty$. The only difference from the short range case $p \geqq q>3$ is the presence of the phase functions $S^{ \pm}$, which modulate the free dynamics in order to take the long range nonlinearities into account. Since the additional factors $\exp \left(i S^{ \pm}\right)$have no contribution to the amplitude of the free dynamics $U(t) \phi_{ \pm}$, the probability density $|u(t)|^{2}$ and the amplitude $|u(t)|$ behave like those of the free dynamics as $t \rightarrow \pm \infty$, as described in part (2) of Corollary 1. A similar property is well known in the Coulomb scattering [18].

By Theorems 1 and 2, the modified wave operators $W_{ \pm}: \phi_{ \pm} \mapsto u(0)$ are well-defined maps from a neighborhood of zero in $H^{0,2}$ to $L^{2}$ or from a neighborhood of the zero in $H^{1,2} \cap H^{0,3}$ to $H^{1,0}$. The Cauchy problem is therefore 
solved so that the asymptotic behavior in time of solutions is described as $(1.10)_{ \pm}$ or $(1.16)_{ \pm}$when the initial data are in the ranges of the modified wave operators. Of course our definition of the modified wave operators is only one of the possible ones, as is in the scattering theory for Schrödinger operators with long range potentials. We should mention here that from a different point of view Flato, Simon \& Taflin [5] constructed modified wave operators in order to solve the Maxwell-Dirac equations globally in time.

We now describe how to find the modified asymptotics for the long range case. By the analogy with the Coulomb case it is reasonable to except that there is a solution $u$ such that $\left\||u(t)|-\left|U(t) \phi_{ \pm}\right|\right\|_{2} \rightarrow 0$ as $t \rightarrow \pm \infty$ for some $\phi_{ \pm}$. By the

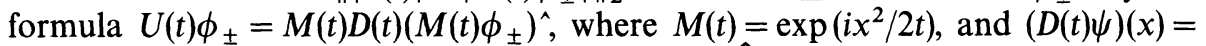
$(i t)^{-1 / 2} \psi\left(t^{-1} x\right)$, we have that $\left\|\left|U(t) \phi_{ \pm}\right|-\left|D(t) \hat{\phi}_{ \pm}\right|\right\|_{2} \rightarrow 0$, as $t \rightarrow \pm \infty$. Hence $\left\||u(t)|-\left|D(t) \hat{\phi}_{ \pm}\right|\right\|_{2} \rightarrow 0$, as $t \rightarrow \pm \infty$. This leads to the observation that $u$ tends to the solutions $u_{ \pm}$of the equations

$$
i \partial_{t} u_{ \pm}+(1 / 2) \partial^{2} u_{ \pm}=\lambda|t|^{-1}\left|\hat{\phi}_{ \pm}\left(t^{-1} x\right)\right|^{2} u_{ \pm}+\mu|t|^{-(p-1) / 2}\left|\hat{\phi}\left(t^{-1} x\right)\right|^{p-1} u_{ \pm}
$$

as $t \rightarrow \pm \infty$. We are thus reduced to looking for approximate solutions for (1.18) which are written explicitly in terms of $\phi_{ \pm}$. This is the reason why the fastors $\exp \left(i S^{ \pm}\right)$appear in front of the free dynamics $U(t) \phi_{ \pm}$in the theorems. In fact, the first candidates $\exp \left(i S^{ \pm}(t)\right) U(t) \phi_{ \pm}$do not give a satisfactory approximation for (1.18). Rather, a good approximation is given by the second candidates

$$
v_{ \pm}(t)=\exp \left(i S^{ \pm}(t)\right) U(t) M(-t) \phi_{ \pm}=\exp \left(i S^{ \pm}(t)\right) M(t) D(t) \hat{\phi}_{ \pm},
$$

which are shown to satisfy (1.18) up to the rate $O\left(|t|^{-2}(\log |t|)^{2}\right)$ in the $L^{2}$-norm as $t \rightarrow \pm \infty$, essentially because of the facts that $\hat{\phi}_{ \pm}$are involved in $v_{ \pm}(t, x)$ in the form $\hat{\phi}_{ \pm}\left(t^{-1} x\right)$ and that the phase factors $\exp \left(i S^{ \pm}\right)$give an appropriate cancellation for the long range potentials $\lambda|t|^{-1}\left|\hat{\phi}_{ \pm}\left(t^{-1} x\right)\right|^{2}+\mu|t|^{-(p-1) / 2}\left|\hat{\phi}_{ \pm}\left(t^{-1} x\right)\right|^{p-1}$. The second candidates $v_{ \pm}$have another advantage that $\left\|v_{ \pm}(t)-\exp \left(i S^{ \pm}(t)\right) U(t) \phi_{ \pm}\right\|_{2} \rightarrow 0$ as $t \rightarrow \pm \infty$. This suggests that we should start with $v_{ \pm}$, construct a solution $u$ of (1.1), and then go back to the first candidates $\exp \left(i S^{ \pm}(t)\right) U(t) \phi_{ \pm}$.

We prove the theorem in the next section. The proof proceeds in three steps. The first step is to solve the integral equations

$$
u(t)=v_{ \pm}(t)+i \int_{t}^{ \pm \infty} U(t-\tau)\left(f(u(\tau))-\left(i \partial_{\tau}+(1 / 2) \partial^{2}\right) v_{ \pm}(\tau)\right) d \tau
$$

in neighborhoods of $t= \pm \infty$ by a contraction method. To this end we define a function space and a suitable metric so that the space is complete and the right-hand sides of (1.19) $)_{ \pm}$are contraction maps of $u$ in the space. That space is constructed as a closed ball centered at $v_{ \pm}$. The proof uses the space-time estimates of the Strichartz type for the propagator $U(t)$. We remark here that the solutions of (1.19) $)_{ \pm}$also satisfy (1.1) near $t= \pm \infty$. The second step is to extend the solutions to the whole real line. We use the well known results on the Cauchy problem described as above to obtain global solutions. In Theorem 2 the restriction $\mu \geqq 0$ comes from obtaining the described a priori estimates from the conservation of the energy. The last step is to prove the estimates described in the theorems.

In the sequel different positive constants might be denoted by the same letter $C$, and if necessary, by $C(*, \ldots, *)$ in order to indicate the dependence on the quantities appearing in parentheses. 


\section{Proof of the Theorems}

In this section we prove Theorems 1 and 2 . In the following we only consider the case $t>0$. The other case is treated analogously. We start by recalling the following lemma concerning the space-time estimates for the integral operator

$$
(G v)(t)=\int_{t}^{\infty} U(t-\tau) v(\tau) d \tau
$$

Lemma $1([2,15,26])$. Let $(q, r)$ satisfy $2 \leqq q \leqq \infty, 4 \leqq r \leqq \infty$, and $1 / 2-1 / q=2 / r$. Let $I=\left(t_{0}, \infty\right)$ with $t_{0}>0$. Then $G: v \mapsto G v$ is a bounded operator from $L^{1}\left(I ; L^{2}\right)$ to $L^{r}\left(I ; L^{q}\right)$ with norm uniformly bounded with respect to $t_{0}$. Moreover, if $v \in L^{1}\left(I ; L^{2}\right)$, then $G v \in C\left(\left[t_{0}, \infty\right) ; L^{2}\right)$.

We next give preliminary estimates for an approximate solution $v_{+}(t)=$ $\exp \left(i S^{+}(t)\right) U(t) M(-t) \phi_{+}=\exp \left(i S^{+}(t)\right) M(t) D(t) \hat{\phi}_{+}$. We define the remainder term $F$ by $F(t)=i \partial_{t} v_{+}(t)+(1 / 2) \partial^{2} v_{+}(t)-f\left(v_{+}(t)\right)$.

Lemma 2. (1) If $\phi_{+} \in H^{0,2}$, then $v_{+} \in C^{1}\left(\mathbb{R}_{+} ; H^{0,-2}\right) \cap C\left(\mathbb{R}_{+} ; L^{2} \cap H^{2,-2}\right)$ and $F \in C\left(\mathbb{R}_{+} ; L^{2}\right)$. Moreover, there exists $C>0$ and $T \geqq 1$ such that for any $\phi_{+} \in H^{0,2}$ and any $t \geqq T$,

$$
\|F(t)\|_{2} \leqq C t^{-2}(\log t)^{2}\left\|\phi_{+}\right\|_{0,2}\left(1+\left\|\phi_{+}\right\|_{0,1}^{2 p-2}\right) .
$$

(2) If $\phi_{+} \in H^{1,2}$, then $v_{+} \in C^{1}\left(\mathbb{R}_{+} ; H^{0,-1}\right) \cap C\left(\mathbb{R}_{+} ; H^{1,0} \cap H^{2,-1}\right)$. If $\phi_{+} \in H^{1,2} \cap$ $H^{0,3}$, then $F \in C\left(\mathbb{R}_{+} ; H^{1,0}\right)$. Moreover, there exists $C>0$ and $T \geqq 1$ such that for any $\phi_{+} \in H^{1,2} \cap H^{0,3}$ and any $t \geqq T$,

$$
\begin{aligned}
\|F(t)\|_{1,0} \leqq & C t^{-2}(\log t)^{2}\left(\left\|\phi_{+}\right\|_{1,2}+\left\|\phi_{+}\right\|_{0,3}\right)\left(1+\left\|\phi_{+}\right\|_{0,1}^{2 p-2}\right) \\
& +C t^{-3}(\log t)^{3}\left\|\phi_{+}\right\|_{0,3}^{2}\left(1+\left\|\phi_{+}\right\|_{0,1}^{3 p-3}\right) .
\end{aligned}
$$

Proof. Let $\phi_{+} \in H^{0,2}$ and let $\tilde{S}(t, x)=x^{2} / 2 t+S^{+}(t, x)$. Then $v_{+}(t, x)=(i t)^{-1 / 2} \times$ $\exp (i \tilde{S}(t, x)) \hat{\phi}\left(t^{-1} x\right)$. By a straightforward calculation we see that $v_{+} \in C^{1}\left(\mathbb{R}_{+} ; H^{0,-2}\right) \cap$ $C\left(\mathbb{R}_{+} ; L^{2} \cap H^{2,-2}\right)$ and $F(t, x)=(i t)^{-1 / 2} \exp (i \tilde{S}(t, x)) \tilde{\phi}\left(t^{-1} x\right)$, where

$$
\begin{aligned}
\tilde{\phi}= & -i(\lambda / 2) t^{-2} \log t \hat{\phi}_{+} \partial^{2}\left|\hat{\phi}_{+}\right|^{2}+i(\mu /(p-3)) t^{-(p+1) / 2} \hat{\phi}_{+} \partial^{2}\left|\hat{\phi}_{+}\right|^{p-1} \\
& -\left.\left.\left(\lambda^{2} / 2\right) t^{-2}(\log t)^{2} \hat{\phi}_{+}|\partial| \hat{\phi}_{+}\right|^{2}\right|^{2}-\left.\left.\left(\mu^{2} / 2(p-3)^{2}\right) t^{-(p-1)} \hat{\phi}_{+}|\partial| \hat{\phi}_{+}\right|^{p-1}\right|^{2} \\
& +(2 \lambda \mu /(p-3)) t^{-(p+1) / 2} \log t \hat{\phi}_{+} \partial\left|\hat{\phi}_{+}\right|^{2} \partial\left|\hat{\phi}_{+}\right|^{p-1} \\
& -i \lambda t^{-2} \log t \partial \hat{\phi}_{+} \partial\left|\hat{\phi}_{+}\right|^{2}+i(2 \mu /(p-3)) t^{-(p+1) / 2} \partial \hat{\phi}_{+} \partial\left|\hat{\phi}_{+}\right|^{p-1} \\
& +(1 / 2) t^{-2} \partial^{2} \hat{\phi}_{+} .
\end{aligned}
$$

By Hölder's inequality and the Gagliardo-Nirenberg inequality of the form $\|\partial \psi\|_{4} \leqq C\left\|\partial^{2} \psi\right\|_{2}^{1 / 2}\|\psi\|_{\infty}^{1 / 2}$ (see [6]), we have

$$
\begin{aligned}
\|F(t)\|_{2} \leqq & C t^{-2} \log t\left(\left\|\partial^{2} \hat{\phi}_{+}\right\|_{2}\left\|\hat{\phi}_{+}\right\|_{\infty}^{2}+\left\|\partial \hat{\phi}_{+}\right\|_{4}^{2}\left\|\hat{\phi}_{+}\right\|_{\infty}\right) \\
& +C t^{-(p+1) / 2}\left(\left\|\partial^{2} \hat{\phi}_{+}\right\|_{2}\left\|\hat{\phi}_{+}\right\|_{\infty}^{p-1}+\left\|\partial \hat{\phi}_{+}\right\|_{4}^{2}\left\|\hat{\phi}_{+}\right\|_{\infty}^{p-2}\right) \\
& +C t^{-2}(\log t)^{2}\left\|\partial \hat{\phi}_{+}\right\|_{4}^{2}\left\|\hat{\phi}_{+}\right\|_{\infty}^{3}+C t^{-(p-1)}\left\|\partial \hat{\phi}_{+}\right\|_{4}^{2}\left\|\hat{\phi}_{+}\right\|_{\infty}^{2 p-3} \\
& +C t^{-(p+1) / 2} \log t\left\|\partial \hat{\phi}_{+}\right\|_{4}^{2}\left\|\hat{\phi}_{+}\right\|_{\infty}^{p-1}+t^{-2}\left\|\partial^{2} \hat{\phi}_{+}\right\|_{2} \\
\leqq & C t^{-2}(\log t)^{2}\left\|\partial^{2} \hat{\phi}_{+}\right\|_{2}\left(\left\|\hat{\phi}_{+}\right\|_{\infty}^{2}+\left\|\hat{\phi}_{+}\right\|_{\infty}^{p-1}+\left\|\hat{\phi}_{+}\right\|_{\infty}^{4}\right.
\end{aligned}
$$




$$
\begin{gathered}
\left.+\left\|\hat{\phi}_{+}\right\|_{\infty}^{2 p-2}+\left\|\hat{\phi}_{+}\right\|_{\infty}^{p}+1\right) \\
\leqq C t^{-2}(\log t)^{2}\left\|\partial^{2} \hat{\phi}_{+}\right\|_{2}\left(1+\left\|\hat{\phi}_{+}\right\|_{\infty}^{2 p-2}\right)
\end{gathered}
$$

for all $t \geqq T$ with $T \geqq 1$ sufficiently large. This proves (2.1) since $\left\|\partial^{2} \hat{\phi}_{+}\right\|_{2} \leqq\left\|\hat{\phi}_{+}\right\|_{0,2}$, $\left\|\hat{\phi}_{+}\right\|_{\infty} \leqq\left\|\phi_{+}\right\|_{0,1}$. Similarly, we have $F \in C\left(\mathbb{R}_{+} ; L^{2}\right)$. We turn to part (2). By a straightforward calculation we see that $v_{+} \in C^{1}\left(\mathbb{R}_{+} ; H^{0,-1}\right) \cap C\left(\mathbb{R}_{+} ; H^{1,0} \cap H^{2,-1}\right)$ for $\phi_{+} \in H^{1,2}$. Let $\phi_{+} \in H^{1,2} \cap H^{0,3}$. Then $\partial F(t, x)=(i t)^{-1 / 2} \exp (i \tilde{S}(t, x)) \tilde{\psi}\left(t^{-1} x\right)$, where

$$
\begin{aligned}
\widetilde{\psi}(y)= & i y \tilde{\phi}(y)-i \lambda t^{-1} \log t \tilde{\phi}(y) \partial\left|\hat{\phi}_{+}\right|^{2}(y) \\
& +i(2 \mu /(p-3)) t^{-(p-1) / 2} \tilde{\phi}(y) \partial\left|\hat{\phi}_{+}\right|^{p-1}(y)+t^{-1} \partial \tilde{\phi}(y) .
\end{aligned}
$$

Accordingly, we decompose $\partial F$ into four terms and denote them as I-IV. We estimate the first term in $L^{2}$ in the same way as above:

$$
\begin{aligned}
\|\mathrm{I}\|_{2}=\|x \tilde{\phi}\|_{2} \leqq & C\left\|x \hat{\phi}_{+}\right\|_{\infty}\left(t^{-2} \log t\left\|\partial^{2}\left|\hat{\phi}_{+}\right|^{2}\right\|_{2}+t^{-(p+1) / 2}\left\|\partial^{2}\left|\hat{\phi}_{+}\right|^{p-1}\right\|_{2}\right. \\
& +t^{-2}(\log t)^{2}\left\|\partial\left|\hat{\phi}_{+}\right|^{2}\right\|_{4}^{2}+t^{-(p-1)}\left\|\partial\left|\hat{\phi}_{+}\right|^{p-1}\right\|_{4}^{2} \\
& +t^{-(p+1) / 2} \log t\left\|\partial \hat{\phi}_{+}\right\|_{4}^{2}\left\|\hat{\phi}_{+}\right\|_{\infty}^{p-1}+t^{-2} \log t\left\|\partial \hat{\phi}_{+}\right\|_{4}^{2} \\
& \left.+t^{-(p+1) / 2}\left\|\partial \hat{\phi}_{+}\right\|_{4}^{2}\left\|\hat{\phi}_{+}\right\|_{\infty}^{p-3}\right)+t^{-2}\left\|x \partial^{2} \hat{\phi}_{+}\right\|_{2} \\
\leqq & C t^{-2}(\log t)^{2}\left\|x \hat{\phi}_{+}\right\|_{\infty}\left\|\partial^{2} \hat{\phi}_{+}\right\|_{2}\left(1+\left\|\hat{\phi}_{+}\right\|_{\infty}^{2 p-3}\right)+t^{-2}\left\|\partial\left(x^{2} \phi_{+}\right)\right\|_{2} \\
\leqq & C t^{-2}(\log t)^{2}\left\|\phi_{+}\right\|_{1,1}\left(1+\left\|\phi_{+}\right\|_{0,2}^{2 p-2}\right)+C t^{-2}\left\|\phi_{+}\right\|_{1,2} \\
\leqq & C t^{-2}(\log t)^{2}\left\|\phi_{+}\right\|_{1,2}\left(1+\left\|\phi_{+}\right\|_{0,2}^{2 p-2}\right)
\end{aligned}
$$

for all $t \geqq T$ with $T \geqq 1$ sufficiently large. The next two terms are estimated as

$$
\begin{aligned}
\|\mathrm{II}+\mathrm{III}\|_{2} & \leqq C\left(t^{-1} \log t\left\|\partial\left|\hat{\phi}_{+}\right|^{2}\right\|_{\infty}+t^{-(p-1) / 2}\left\|\partial\left|\hat{\phi}_{+}\right|^{p-1}\right\|_{\infty}\right)\|F(t)\|_{2} \\
& \leqq C t^{-3}(\log t)^{3}\left\|\phi_{+}\right\|_{0,2}\left(1+\left\|\phi_{+}\right\|_{0,1}^{p-2}\right)\|F(t)\|_{2} \\
& \leqq C t^{-3}(\log t)^{3}\left\|\phi_{+}\right\|_{0,2}^{3}\left(1+\left\|\phi_{+}\right\|_{0,1}^{3 p-4}\right) \\
& \leqq C t^{-3}(\log t)^{3}\left\|\phi_{+}\right\|_{0,3}^{2}\left(1+\left\|\phi_{+}\right\|_{0,1}^{3 p-3}\right),
\end{aligned}
$$

where we have used (2.1) and the inequalities $\left\|\partial\left|\hat{\phi}_{+}\right|^{q-1}\right\|_{\infty} \leqq C\left\|\partial \hat{\phi}_{+}\right\|_{\infty}\left\|\hat{\phi}_{+}\right\|_{\infty}^{q-2} \leqq$ $C\left\|\phi_{+}\right\|_{0,2}\left\|\phi_{+}\right\|_{0,1}^{q-2}$ for $q \geqq 2$ and $\left\|\phi_{+}\right\|_{0,2} \leqq\left\|\phi_{+}\right\|_{0,3}^{2 / 3}\left\|\phi_{+}\right\|_{2}^{1 / 3}$. For the last term, we have

$$
\begin{aligned}
\|\mathrm{IV}\|_{2}= & t^{-1}\|\partial \tilde{\phi}\|_{2} \\
\leqq & C t^{-3} \log t\left(\left\|\partial \hat{\phi}_{+}\right\|_{6}^{3}+\left\|\partial^{2} \hat{\phi}_{+}\right\|_{3}\left\|\partial \hat{\phi}_{+}\right\|_{6}\left\|\hat{\phi}_{+}\right\|_{\infty}+\left\|\partial^{3} \hat{\phi}_{+}\right\|_{2}\left\|\hat{\phi}_{+}\right\|_{\infty}^{2}\right) \\
& +C t^{-(p+3) / 2}\left(\left\|\partial \hat{\phi}_{+}\right\|_{6}^{3}\left\|\hat{\phi}_{+}\right\|_{\infty}^{p-3}+\left\|\partial^{2} \hat{\phi}_{+}\right\|_{3}\left\|\partial \hat{\phi}_{+}\right\|_{6}\left\|\hat{\phi}_{+}\right\|_{\infty}^{p-2}\right. \\
& \left.+\left\|\partial^{3} \hat{\phi}_{+}\right\|_{2}\left\|\hat{\phi}_{+}\right\|_{\infty}^{p-1}\right) \\
& +C t^{-3}(\log t)^{2}\left(\left\|\partial \hat{\phi}_{+}\right\|_{6}^{3}\left\|\hat{\phi}_{+}\right\|_{\infty}^{2}+\left\|\partial^{2} \hat{\phi}_{+}\right\|_{3}\left\|\partial \hat{\phi}_{+}\right\|_{6} \|_{+} \hat{\phi}_{+\infty}^{3}\right) \\
& +C t^{-p}\left(\left\|\partial \hat{\phi}_{+}\right\|_{6}^{3}\left\|\hat{\phi}_{+}\right\|_{\infty}^{2 p-4}+\left\|\partial^{2} \hat{\phi}_{+}\right\|_{3}\left\|\partial \hat{\phi}_{+}\right\|_{6}\left\|\hat{\phi}_{+}\right\|^{2 p-3}\right) \\
& +C t^{-(p+3) / 2} \log t\left(\left\|\partial \hat{\phi}_{+}\right\|_{6}^{3}\left\|\hat{\phi}_{+}\right\|_{\infty}^{p-2}+\left\|\partial^{2} \hat{\phi}_{+}\right\|_{3}\left\|\partial \hat{\phi}_{+}\right\|_{6} \|_{+} \hat{\phi}_{\infty}^{p-1}\right) \\
& +C t^{-3}\left\|\partial^{3} \hat{\phi}_{+}\right\|_{2}
\end{aligned}
$$




$$
\begin{aligned}
\leqq & C t^{-3}(\log t)^{2}\left\|\partial^{2} \hat{\phi}_{+}\right\|_{2}\left(\left\|\hat{\phi}_{+}\right\|_{\infty}^{2}+\left\|\hat{\phi}_{+}\right\|_{\infty}^{p-1}+\left\|\hat{\phi}_{+}\right\|_{\infty}^{4}+\left\|\hat{\phi}_{+}\right\|_{\infty}^{2 p-2}\right. \\
& \left.+\left\|\hat{\phi}_{+}\right\|_{\infty}^{p}+1\right) \\
\leqq & C t^{-3}(\log t)^{2}\left\|\hat{\phi}_{+}\right\|_{0,3}\left(1+\left\|\hat{\phi}_{+}\right\|_{0,1}^{2 p-2}\right),
\end{aligned}
$$

where we have used Hölder's inequality and the Gagliardo-Nirenberg inequalities (see [6]) $\|\partial \psi\|_{6} \leqq C\left\|\partial^{3} \psi\right\|_{2}^{1 / 3}\|\psi\|_{\infty}^{2 / 3},\left\|\partial^{2} \psi\right\|_{3} \leqq C\left\|\partial^{3} \psi\right\|_{2}^{2 / 3}\|\psi\|_{\infty}^{1 / 3}$. Collecting these estimates, we obtain (2.2). Similarly, we have $F \in C\left(\mathbb{R}_{+} ; H^{1},{ }^{0}\right)$. Q.E.D.

For $\alpha \in(1 / 2,1), R>0, T \geqq 1$, and $\phi_{+} \in H^{0,2}$, we introduce

$$
\begin{aligned}
X=X_{R}^{\alpha}(T)= & \left\{u \in C\left([T, \infty) ; L^{2}\right) \cap L^{4}\left(T, \infty ; L^{\infty}\right) ;\right. \\
& \left.\sup _{t \geqq T} t^{\alpha}\left(\left\|u(t)-v_{+}(t)\right\|_{2}+\left(\int_{t}^{\infty}\left\|u(\tau)-v_{+}(\tau)\right\|_{\infty}^{4} d \tau\right)^{1 / 4}\right) \leqq R\right\}
\end{aligned}
$$

and define on $X$ the metric $d\left(u_{1}, u_{2}\right)=\|\| u_{1}-u_{2} \|_{X}$, where

$$
\|\| u \|=\sup _{t \geqq T} t^{\alpha}\left(\|u(t)\|_{2}+\left(\int_{t}^{\infty}\|u(\tau)\|_{\infty}^{4} d \tau\right)^{1 / 4}\right) .
$$

With this metric $X$ becomes a complete space. We define the map $J$ by

$$
\begin{aligned}
(J u)(t) & =v_{+}(t)+i \int_{t}^{\infty} U(t-\tau)\left(f(u(\tau))-\left(i \partial_{\tau}+(1 / 2) \partial^{2}\right) v_{+}(\tau)\right) d \tau \\
& =v_{+}(t)+i \int_{t}^{\infty} U(t-\tau)\left(f(u(\tau))-f\left(v_{+}(\tau)\right)-F(\tau)\right) d \tau .
\end{aligned}
$$

Proof of Theorem 1. Let $\phi_{+} \in H^{0,2}$ and let $u \in X_{R}^{\alpha}(T)$. We have

$$
\begin{aligned}
\left(\int_{t}^{\infty}\left\|v_{+}(\tau)\right\|_{\infty}^{4} d \tau\right)^{1 / 4} & =\left(\int_{t}^{\infty} \tau^{-2}\left\|\hat{\phi}_{+}\right\|_{\infty}^{4} d \tau\right)^{1 / 4}=t^{-1 / 4}\left\|\hat{\phi}_{+}\right\|_{\infty} \\
\left(\int_{t}^{\infty}\|u(\tau)\|_{\infty}^{4} d \tau\right)^{1 / 4} & \leqq\left(\int_{t}^{\infty}\left\|u(\tau)-v_{+}(\tau)\right\|_{\infty}^{4} d \tau\right)^{1 / 4}+\left(\int_{t}^{\infty}\left\|v_{+}(\tau)\right\|_{\infty}^{4} d \tau\right)^{1 / 4} \\
& \leqq R t^{-\alpha}+\left\|\hat{\phi}_{+}\right\|_{\infty} t^{-1 / 4}
\end{aligned}
$$

We prove that $J$ maps $X_{R}^{\alpha}(T)$ into itself and is a contraction in the metric on $X$ if $T$ is sufficiently large and $\left\|\hat{\phi}_{+}\right\|_{\infty}$ is sufficiently small. By Lemma 1 and (2.3),

$$
\begin{aligned}
& \left\|(J u)(t)-v_{+}(t)\right\|_{2}+\left(\int_{t}^{\infty}\left\|(J u)(\tau)-v_{+}(\tau)\right\|_{\infty}^{4} d \tau\right)^{1 / 4} \\
& \leqq C \int_{t}^{\infty}\left\|f(u(\tau))-f\left(v_{+}(\tau)\right)\right\|_{2} d \tau+C \int_{t}^{\infty}\|F(\tau)\|_{2} d \tau .
\end{aligned}
$$

By Hölder's inequality, (2.4), and (2.5),

$$
\begin{aligned}
& \int_{t}^{\infty}\left\|f(u(\tau))-f\left(v_{+}(\tau)\right)\right\|_{2} d \tau \\
& \quad \leqq C \int_{t}^{\infty}\left(\|u(\tau)\|_{\infty}^{2}+\left\|v_{+}(\tau)\right\|_{\infty}^{2}+\|u(\tau)\|_{\infty}^{p-1}+\left\|v_{+}(\tau)\right\|_{\infty}^{p-1}\right)\left\|u(\tau)-v_{+}(\tau)\right\|_{2} d \tau
\end{aligned}
$$




$$
\begin{aligned}
\leqq & C\left(\left(\int_{t}^{\infty}\|u(\tau)\|_{\infty}^{4} d \tau\right)^{1 / 2}+\left(\int_{t}^{\infty}\left\|v_{+}(\tau)\right\|_{\infty}^{4} d \tau\right)^{1 / 2}\right)\left(\int_{t}^{\infty}\left\|u(\tau)-v_{+}(\tau)\right\|_{2}^{2} d \tau\right)^{1 / 2} \\
& +C\left(\left(\int_{t}^{\infty}\|u(\tau)\|_{\infty}^{4} d \tau\right)^{(p-1) / 4}+\left(\int_{t}^{\infty}\left\|v_{+}(\tau)\right\|_{\infty}^{4} d \tau\right)^{(p-1) / 4}\right) \\
& \cdot\left(\int_{t}^{\infty}\left\|u(\tau)-v_{+}(\tau)\right\|_{2}^{4 /(5-p)} d \tau\right)^{(5-p) / 4} \\
\leqq & C R\left(R^{2} t^{-2 \alpha}+\left\|\hat{\phi}_{+}\right\|_{\infty}^{2} t^{-1 / 2}\right)\left(\int_{t}^{\infty} \tau^{-2 \alpha} d \tau\right)^{1 / 2} d \tau \\
& +C R\left(R^{p-1} t^{-(p-1) \alpha}+\left\|\hat{\phi}_{+}\right\|_{\infty}^{p-1} t^{-(p-1) / 4}\right)\left(\int_{t}^{\infty} \tau^{-4 \alpha /(5-p)} d \tau\right)^{(5-p) / 4} \\
\leqq & C R t^{-\alpha}\left(R^{2} t^{1 / 2-2 \alpha}+R^{p-1} t^{(5-p) / 4-(p-1) \alpha}+\left\|\hat{\phi}_{+}\right\|_{\infty}^{2}+\left\|\hat{\phi}_{+}\right\|_{\infty}^{p-1} t^{-(p-3) / 2}\right) .
\end{aligned}
$$

We note here that $1 / 2-2 \alpha<-1 / 2,(5-p) / 4-(p-1) \alpha<-1 / 2$. By (2.1),

$$
\int_{t}^{\infty}\|F(\tau)\|_{2} d \tau \leqq C t^{-1}(\log t)^{2}\left\|\phi_{+}\right\|_{0,2}^{2}\left(1+\left\|\phi_{+}\right\|_{0,1}^{2 p-2}\right)
$$

for all $t \geqq T$ with $T \geqq 1$ large enough. By (2.6), (2.7), and (2.8),

$$
\left\|J u-v_{+}\right\|_{X} \leqq C R\left\|\hat{\phi}_{+}\right\|_{\infty}^{2}
$$

for $T \geqq 1$ large enough. In the same way as above, for $u_{1}, u_{2} \in X_{R}^{\alpha}(T)$,

$$
\begin{aligned}
& \left\|\left(J u_{1}-J u_{2}\right)(t)\right\|_{2}+\left(\int_{t}^{\infty}\left\|\left(J u_{1}-J u_{2}\right)(\tau)\right\|_{\infty}^{4} d \tau\right)^{1 / 4} \\
& \quad \leqq C t^{-\alpha}\left(R^{2} t^{1 / 2-2 \alpha}+R^{p-1} t^{(5-p) / 4-(p-1) \alpha}+\left\|\hat{\phi}_{+}\right\|_{\infty}^{2}+\left\|\hat{\phi}_{+}\right\|_{\infty}^{p-1} t^{-(p-3) / 2}\right) \\
& \quad \cdot \sup _{\tau} \tau^{\alpha}\left\|\left(u_{1}-u_{2}\right)(\tau)\right\|_{2},
\end{aligned}
$$

which leads to

$$
\|\| J u_{1}-J u_{2}\left\|_{X} \leqq C\right\| \hat{\phi}_{+}\left\|_{\infty}^{2}\right\| u_{1}-u_{2} \|_{X}
$$

for $T \geqq 1$ large enough. We see from (2.9) and (2.10) that if $\left\|\hat{\phi}_{+}\right\|_{\infty}$ is sufficiently small, $J$ has a unique fixed point $u$ in $X_{R}^{\alpha}(T)$. Therefore $u$ solves the integral equation

$$
u(t)=v_{+}(t)+i \int_{t}^{\infty} U(t-\tau)\left(f(u(\tau))-\left(i \partial_{\tau}+(1 / 2) \partial^{2}\right) v_{+}(\tau)\right) d \tau
$$

for all $t \geqq T$. Let $t>t_{0} \geqq T$. Using (2.11), we obtain

$$
\begin{aligned}
U(-t)\left(u(t)-v_{+}(t)\right)= & U\left(-t_{0}\right)\left(u\left(t_{0}\right)-v_{+}\left(t_{0}\right)\right) \\
& +i \int_{t_{0}}^{t} U(-\tau)\left(f(u(\tau))-\left(i \partial_{\tau}+(1 / 2) \partial^{2}\right) v_{+}(\tau)\right) d \tau .
\end{aligned}
$$

Noting that

$$
v_{+}(t)=U\left(t-t_{0}\right) v_{+}\left(t_{0}\right)-i \int_{t_{0}}^{t} U(t-\tau)\left(i \partial_{\tau}+(1 / 2) \partial^{2}\right) v_{+}(\tau) d \tau
$$


we deduce from (2.12) that $u$ solves the integral equation

$$
u(t)=U\left(t-t_{0}\right) u\left(t_{0}\right) u\left(t_{0}\right)-i \int_{t_{0}}^{t} U(t-\tau) f(u(\tau)) d \tau .
$$

It is well known that (2.13) has a unique global solution in $C\left(\mathbb{R} ; L^{2}\right) \cap L_{\text {loc }}^{4}\left(\mathbb{R} ; L^{\infty}\right)$ and therefore the solution $u$ of $(2.11)$ extends to all times and satisfies (2.13) for all $t \in \mathbb{R}$. By a standard argument, $u$ satisfies $(1.1)$ in $H^{-2,0}$ for almost all $t \in \mathbb{R}$. We now prove $(1.8)_{+}$and $(1.9)_{+}$. By the inequality $\left|\exp \left(-i x^{2} / 2 t\right)-1\right| \leqq x^{2} / 2|t|$, we have

$$
\begin{aligned}
& \left\|u(t)-\exp \left(i S^{+}(t)\right) U(t) \phi_{+}\right\|_{2} \\
& \quad \leqq\left\|u(t)-\exp \left(i S^{+}(t)\right) U(t) M(-t) \phi_{+}\right\|_{2}+\left\|\exp \left(i S^{+}(t)\right) U(t)\left(M(-t) \phi_{+}-\phi_{+}\right)\right\|_{2} \\
& \quad \leqq C t^{-\alpha}+\left\|M(-t) \phi_{+}-\phi_{+}\right\|_{2} \leqq C t^{-\alpha}+t^{-1}\left\|\phi_{+}\right\|_{0,2} .
\end{aligned}
$$

This proves $(1.8)_{+}$. By the inequalities $\|U(t) \psi\|_{\infty} \leqq t^{-1 / 2}\|\psi\|_{1}$ and $\left|\exp \left(-i x^{2} / 2 t\right)-1\right| \leqq 2|x|^{3 / 2-\varepsilon}|t|^{-3 / 4+\varepsilon / 2}$ for $0<\varepsilon<3 / 2$, we have

$$
\begin{aligned}
& \left\|u(t)-\exp \left(i S^{+}(t)\right) U(t) \phi_{+}\right\|_{\infty} \\
& \quad \leqq\left\|u(t)-\exp \left(i S^{+}(t)\right) U(t) M(-t) \phi_{+}\right\|_{\infty}+C t^{-5 / 4+\varepsilon / 2}\left\|x^{3 / 2-\varepsilon} \phi_{+}\right\|_{1},
\end{aligned}
$$

and therefore

$$
\begin{aligned}
& \left(\int_{t}^{\infty}\left\|u(\tau)-\exp \left(i S^{+}(\tau)\right) U(\tau) \phi_{+}\right\|_{\infty}^{4} d \tau\right)^{1 / 4} \\
& \quad \leqq C t^{-\alpha}+C t^{-1+\varepsilon / 2}\left\|x^{3 / 2-\varepsilon} \phi_{+}\right\|_{1} \leqq C t^{-\alpha}+C(\varepsilon) t^{-1+\varepsilon / 2}\left\|\phi_{+}\right\|_{0,2} .
\end{aligned}
$$

Choosing $\varepsilon>0$ so that $\varepsilon<2(1-\alpha)$, we have $(1.9)_{+}$. We finally prove the uniqueness. Let $u_{1}$ and $u_{2}$ be solutions of (1.1) satisfying $(1.8)_{+}$and $(1.9)_{+}$. In the same way as in (2.14)-(2.16) we have $u_{1}, u_{2} \in X_{R}^{\alpha}(T)$ for some $R>0, T \geqq 1$. In the same way as in the derivation of (1.1) from (2.11) we see that $u_{1}$ and $u_{2}$ solve (2.11). By the uniqueness of solutions of (2.11) we have $u_{1}(t)=u_{2}(t)$ for all $t \geqq T$. By the uniqueness of solutions of (2.13), we have $u_{1}(t)=u_{2}(t)$ for all $t \in \mathbb{R}$. Q.E.D.

Proof of Corollary 1. Let $\phi_{+}$and $u$ be as in Theorem 1. By $(1.8)_{+}$,

$$
\begin{aligned}
& \left\|u(t)-\exp \left(i S_{0}^{+}(t)\right) U(t) \phi_{+}\right\|_{2} \\
& \quad \leqq\left\|u(t)-\exp \left(i S^{+}(t)\right) U(t) \phi_{+}\right\|_{2}+\left\|\left(\exp \left(i S^{+}(t)\right)-\exp \left(i S_{0}^{+}(t)\right)\right) U(t) \phi_{+}\right\|_{2} \\
& \quad \leqq C t^{-\alpha}+\left\|\exp \left(i\left(S^{+}(t)-S_{0}^{+}(t)\right)\right)-1\right\|_{\infty}\left\|U(t) \phi_{+}\right\|_{2} \\
& \quad \leqq C t^{-\alpha}+\left\|S^{+}(t)-S_{0}^{+}(t)\right\|_{\infty}\left\|\phi_{+}\right\|_{2} \leqq C t^{-\alpha}+C|\mu| t^{-(p-3) / 2}\left\|\hat{\phi}_{+}\right\|_{\infty}^{p-1}\left\|\phi_{+}\right\|_{2} .
\end{aligned}
$$

This proves $(1.10)_{+}$. By $(1.8)_{+}$and the conservation law of the $L^{2}$-norm $\|u(t)\|_{2}=\|u(0)\|_{2}$ for all $t \in \mathbb{R}$, we have

$$
\begin{aligned}
\left|\|u(0)\|_{2}-\left\|\phi_{+}\right\|_{2}\right| & =\left|\|u(t)\|_{2}-\left\|\exp \left(i S^{+}(t)\right) U(t) \phi_{+}\right\|_{2}\right| \\
& \leqq\left\|u(t)-\exp \left(i S^{+}(t)\right) U(t) \phi_{+}\right\|_{2} \rightarrow 0 \text { as } t \rightarrow+\infty
\end{aligned}
$$

and therefore $\|u(t)\|_{2}=\|u(0)\|_{2}=\left\|\phi_{+}\right\|_{2}$ for all $t \in \mathbb{R}$. Then, by $(1.8)_{+}$

$$
\begin{aligned}
& \left\||u(t)|^{2}-\left|U(t) \phi_{+}\right|^{2}\right\|_{1} \\
& \quad=\left\||u(t)|^{2}-\left|\exp \left(i S^{+}(t)\right) U(t) \phi_{+}\right|^{2}\right\|_{1}
\end{aligned}
$$




$$
\begin{aligned}
& \leqq\left(\|u(t)\|_{2}+\left\|\exp \left(i S^{+}(t)\right) U(t) \phi_{+}\right\|_{2}\right)\left\|u(t)-\exp \left(i S^{+}(t)\right) U(t) \phi_{+}\right\|_{2} \\
& =2\left\|\phi_{+}\right\|_{2}\left\|u(t)-\exp \left(i S^{+}(t)\right) U(t) \phi_{+}\right\|_{2}=O\left(t^{-\alpha}\right)
\end{aligned}
$$

as $t \rightarrow+\infty$. This proves $(1.11)_{+}$. Similarly, denoting by $(\cdot, \cdot)$ the $L^{2}$-scalar product, we get

$$
\begin{aligned}
\left\||u(t)|-\left|U(t) \phi_{+}\right|\right\|_{2}^{2} & =\left\|U(t) \phi_{+}\right\|_{2}^{2}-\|u(t)\|_{2}^{2}+2\left(|u(t)|,|u(t)|-\left|U(t) \phi_{+}\right|\right) \\
& =2\left(|u(t)|,|u(t)|-\left|\exp \left(i S^{+}(t)\right) U(t) \phi_{+}\right|\right) \\
& \leqq 2\|u(t)\|_{2}\left\|u(t)-\exp \left(i S^{+}(t)\right) U(t) \phi_{+}\right\|_{2}=O\left(t^{-\alpha}\right)
\end{aligned}
$$

as $t \rightarrow+\infty$. This proves $(1.12)_{+}$. We have

$$
\left\|\exp \left(i S^{+}(t)\right) U(t) \phi_{+}\right\|_{\infty}=\left\|U(t) \phi_{+}\right\|_{\infty} \leqq t^{-1 / 2}\left\|\phi_{+}\right\|_{1} \leqq t^{-1 / 2}\left\|\phi_{+}\right\|_{0,1},
$$

so that by $(1.9)_{+}$,

$$
\begin{aligned}
\left(\int_{t}^{\infty}\|u(\tau)\|_{\infty}^{4} d \tau\right)^{1 / 4} \leqq & \left(\int_{t}^{\infty}\left\|u(\tau)-\exp \left(i S^{+}(\tau)\right) U(\tau) \phi_{+}\right\|_{\infty}^{4} d \tau\right)^{1 / 4} \\
& +\left(\int_{t}^{\infty}\left\|\exp \left(i S^{+}(\tau)\right) U(\tau) \phi_{+}\right\|_{\infty}^{4} d \tau\right)^{1 / 4} \leqq C t^{-\alpha}+C t^{-1 / 4}\left\|\phi_{+}\right\|_{0,1} .
\end{aligned}
$$

This proves $(1.13)_{+} \cdot$ Q.E.D.

For $\alpha \in(1 / 2,1), R>0, T \geqq 1$, and $\phi_{+} \in H^{1,2} \cap H^{0,3}$, we introduce

$$
Y=Y_{R}^{\alpha}(T)=\left\{u \in X_{R}^{\alpha}(T) ; \partial u \in X_{R}^{\alpha}(T),\left\|u-v_{+}\right\|_{X}+\left\|\partial u-\partial v_{+} \mid\right\|_{X} \leqq R\right\}
$$

and define on $Y$ the metric $d\left(u_{1}, u_{2}\right)=\left\|u_{1}-u_{2}\right\|_{Y}$, where $\|u\|_{Y}=\|u\|_{X}+\|\partial u\|_{X}$. Proof of Theorem 2. Let $\phi_{+} \in H^{1,2} \cap H^{0,3}$ and let $u \in Y_{R}^{\alpha}(T)$. We have $\partial v_{+}(t, x)=$ $(i t)^{-1 / 2} \exp (i \tilde{S}(t, x)) \psi_{+}\left(t^{-1} x\right)$, where

$$
\begin{aligned}
& \begin{aligned}
\begin{array}{l}
\psi_{+}(y)= \\
\end{array} & i y \hat{\phi}_{+}(y)-i \lambda t^{-1} \log t \hat{\phi}_{+}(y) \partial\left|\hat{\phi}_{+}\right|^{2}(y) \\
& +i(2 \mu /(p-3)) t^{-(p+1) / 2} \hat{\phi}_{+}(y) \partial\left|\hat{\phi}_{+}\right|^{p-1}(y)+t^{-1} \partial \hat{\phi}_{+}(y),
\end{aligned} \\
& \text { so that }
\end{aligned}
$$

$$
\begin{aligned}
\left\|\partial v_{+}(t)\right\|_{\infty} \leqq & C t^{-1 / 2}\left(\left\|x \hat{\phi}_{+}\right\|_{\infty}+t^{-1} \log t\left\|\hat{\phi}_{+}\right\|_{\infty}^{2}\left\|\partial \hat{\phi}_{+}\right\|_{\infty}\right. \\
& +t^{-(p+1) / 2}\left\|\hat{\phi}_{+}\right\|_{\infty}^{p-1}\left\|\partial \hat{\phi}_{+}\right\|_{\infty}+t^{-1}\left\|\partial \hat{\phi}_{+}\right\|_{\infty} \\
\leqq & C t^{-1 / 2}\left\|x \hat{\phi}_{+}\right\|_{\infty}+C t^{-3 / 2} \log t\left\|x \phi_{+}\right\|_{1}\left(1+\left\|\hat{\phi}_{+}\right\|_{\infty}^{p-1}\right) \\
\leqq & C t^{-1 / 2}\left\|x \hat{\phi}_{+}\right\|_{\infty}+C t^{-3 / 2} \log t M_{1}\left(\phi_{+}\right)
\end{aligned}
$$

for all $t \geqq T$ with $T \geqq 1$ large enough, where $M_{1}\left(\phi_{+}\right)=\left\|\phi_{+}\right\|_{0,2}\left(1+\left\|\phi_{+}\right\|_{0,1}^{p-1}\right)$. Then, for all $t \geqq T$,

$$
\left(\int_{t}^{\infty}\|\partial u(\tau)\|_{\infty}^{4} d \tau\right)^{1 / 4} \leqq\left(\int_{t}^{\infty}\left\|\partial u(\tau)-\partial v_{+}(\tau)\right\|_{\infty}^{4} d \tau\right)^{1 / 4}+\left(\int_{t}^{\infty}\left\|\partial v_{+}(\tau)\right\|_{\infty}^{4} d \tau\right)^{1 / 4}
$$

Moreover,

$$
\leqq R t^{-\alpha}+C\left\|x \hat{\phi}_{+}\right\|_{\infty} t^{-1 / 4}+C M_{1}\left(\phi_{+}\right) t^{-5 / 4} \log t .
$$

$$
\begin{aligned}
\|u(t)\|_{\infty} & \leqq\left\|u(t)-v_{+}(t)\right\|_{\infty}+\left\|v_{+}(t)\right\|_{\infty} \\
& \leqq C\left\|u(t)-v_{+}(t)\right\|_{1,0}+\left\|\hat{\phi}_{+}\right\|_{\infty} t^{-1 / 2} \leqq C R t^{-\alpha}+\left\|\hat{\phi}_{+}\right\|_{\infty} t^{-1 / 2}
\end{aligned}
$$


By (2.19),

$$
\begin{aligned}
\int_{t}^{\infty} \| & f(u(\tau))-f\left(v_{+}(\tau)\right) \|_{2} d \tau \\
\leqq & C \int_{t}^{\infty}\left(R^{2} \tau^{-2 \alpha}+R^{p-1} \tau^{-(p-1) \alpha}+\left\|\hat{\phi}_{+}\right\|_{\infty}^{2} \tau^{-1}+\left\|\hat{\phi}_{+}\right\|_{\infty}^{p-1} t^{-(p-1) / 2}\right) \\
& \cdot\left\|u(\tau)-v_{+}(\tau)\right\|_{2} d \tau \\
\leqq & C R\left(R^{2} t^{1-3 \alpha}+R^{p-1} t^{1-p \alpha}+\left\|\hat{\phi}_{+}\right\|_{\infty}^{2} t^{-\alpha}+\left\|\hat{\phi}_{+}\right\|_{\infty}^{p-1} t^{-(p-3) / 2-\alpha}\right)
\end{aligned}
$$

Again by (2.19),

$$
\begin{aligned}
&\left\|\partial\left(f(u(t))-f\left(v_{+}(t)\right)\right)\right\|_{2} \\
& \leqq C\left(\|u(t)\|_{\infty}^{p-2}+\left\|v_{+}(t)\right\|_{\infty}^{p-2}\right)\|\partial u(t)\|_{\infty}\left\|u(t)-v_{+}(t)\right\|_{2} \\
&+C\left\|v_{+}(t)\right\|_{\infty}^{p-1}\left\|u(t)-v_{+}(t)\right\|_{2} \\
&+C\left(\|u(t)\|_{\infty}+\left\|v_{+}(t)\right\|_{\infty}\right)\|\partial u(t)\|_{\infty}\left\|u(t)-v_{+}(t)\right\|_{2} \\
&+C\left\|v_{+}(t)\right\|_{\infty}^{2}\left\|u(t)-v_{+}(t)\right\|_{2} \\
& \leqq C\left(R^{p-2} t^{-(p-2) \alpha}+R t^{-\alpha}+\left\|\hat{\phi}_{+}\right\|_{\infty}^{p-2} t^{-(p-2) / 2}+\left\|\hat{\phi}_{+}\right\|_{\infty} t^{-1 / 2}\right) R t^{-\alpha}\|\partial u(t)\|_{\infty} \\
&+C\left(\left\|\hat{\phi}_{+}\right\|_{\infty}^{p-1} t^{-(p-1) / 2}+\left\|\hat{\phi}_{+}\right\|_{\infty}^{2} t^{-1}\right) R t^{-\alpha},
\end{aligned}
$$

which together with (2.18) implies

$$
\begin{aligned}
\int_{t}^{\infty} \| \partial( & \left.f(u(\tau))-f\left(v_{+}(\tau)\right)\right) \|_{2} d \tau \\
\leqq & C R\left(\int_{t}^{\infty}\|\partial u(\tau)\|_{\infty}^{4} d \tau\right)^{1 / 4}\left(R^{p-2}\left(\int_{t}^{\infty} \tau^{-4(p-1) \alpha / 3} d \tau\right)^{3 / 4}+R\left(\int_{t}^{\infty} \tau^{-8 \alpha / 3} d \tau\right)^{3 / 4}\right. \\
& \left.+\left\|\hat{\phi}_{+}\right\|_{\infty}^{p-2}\left(\int_{t}^{\infty} \tau^{-4 \alpha / 3-2(p-2) / 3} d \tau\right)^{3 / 4}+\left\|\hat{\phi}_{+}\right\|_{\infty}\left(\int_{t}^{\infty} \tau^{-4 \alpha / 3-2 / 3} d \tau\right)^{3 / 4}\right) \\
& +C\left\|\hat{\phi}_{+}\right\|_{\infty}^{p-1} R t^{-\alpha-(p-3) / 2}+C\left\|_{+}\right\|_{\infty}^{2} R t^{-\alpha} \\
\leqq & C R\left(R t^{-\alpha}+\left\|x \hat{\phi}_{+}\right\|_{\infty} t^{-1 / 4}+M_{1}\left(\phi_{+}\right) t^{-5 / 4} \log t\right) \\
& +\left(R^{p-2} t^{3 / 4-(p-1) \alpha}+R t^{3 / 4-2 \alpha}+\left\|\hat{\phi}_{+}\right\|_{\infty}^{p-2} t^{1 / 4-\alpha-(p-3) / 2}+\left\|\hat{\phi}_{+}\right\|_{\infty} t^{1 / 4-\alpha}\right) \\
& +C\left\|\hat{\phi}_{+}\right\|_{\infty}^{p-1} R t^{-\alpha-(p-3) / 2}+C\left\|_{+}\right\|_{\infty}^{2} R t^{-\alpha} \\
\leqq & C R^{2}\left(1+R^{p-2}+\left\|\hat{\phi}_{+}\right\|_{\infty}^{p-2}\right) t^{-\alpha-1 / 4}+C R\left(1+R^{p-2}\right)\left\|x \hat{\phi}_{+}\right\|_{\infty} t^{1 / 2-2 \alpha} \\
& +C R\left\|\hat{\phi}_{+}\right\|_{\infty}^{p-2}\left(\left\|x \hat{\phi}_{+}\right\|_{\infty}+\left\|\hat{\phi}_{+}\right\|_{\infty}\right) t^{-\alpha-(p-3) / 2} \\
& +C R\left\|\hat{\phi}_{+}\right\|_{\infty}\left(\left\|\hat{\phi}_{+}\right\|_{\infty}+\left\|x \hat{\phi}_{+}\right\|_{\infty}\right) t^{-\alpha} \\
& +C R M_{1}\left(\phi_{+}\right)\left(1+R^{p-2}+\left\|\hat{\phi}_{+}\right\|_{\infty}^{p-2}\right) t^{-1-\alpha} \log t
\end{aligned}
$$

for all $t \geqq T$ with $T \geqq 1$ large enough. By (2.2),

$$
\int_{t}^{\infty}\|\partial F(\tau)\|_{2} d \tau \leqq C t^{-1}(\log t)^{2} M_{2}\left(\phi_{+}\right)
$$


for all $t \geqq T$ with $T \geqq 1$ large enough, where

$$
M_{2}\left(\phi_{+}\right)=\left(\left\|\phi_{+}\right\|_{1,2}+\left\|\phi_{+}\right\|_{0,3}\right)\left(1+\left\|\phi_{+}\right\|_{0,1}^{2 p-2}\right)+\left\|\phi_{+}\right\|_{0,3}^{2}\left(1+\left\|\phi_{+}\right\|_{0,1}^{3 p-3}\right) .
$$

It follows from (2.8), (2.20), (2.21), and (2.22) that if $T \geqq 1$ is large enough, $J u-v_{+} \in C\left([T, \infty) ; H^{1,0}\right) \cap L^{4}\left(T, \infty ; W^{1, \infty}\right)$ and for all $t \geqq T$,

$$
\begin{gathered}
\left\|\left(J u-v_{+}\right)(t)\right\|_{1,0}+\left(\int_{t}^{\infty}\left\|\left(J u-v_{+}\right)(\tau)\right\|_{W^{1, x}}^{4} d \tau\right)^{1 / 4} \\
\leqq C R\|\hat{\phi}\|_{\infty}\left(\left\|\hat{\phi}_{+}\right\|_{\infty}+\left\|x \hat{\phi}_{+}\right\|_{\infty}\right) t^{-\alpha} .
\end{gathered}
$$

We now distinguish between two cases: (1) $p<5$. (2) $p \geqq 5$.

(1) When $p<5$, we already know that $J$ has a unique fixed point $u$ in $X_{R}^{\alpha}(T)$. From the argument above we show that if $\phi_{+} \in H^{1,2} \cap H^{0,3}$, then the solution $u$ belongs to $Y_{R}^{\alpha}(T)$.

(2) When $p \geqq 5$, in the same way as in the derivation of (2.23) we have

$$
\left\|J u_{1}-J u_{2}\right\|_{Y} \leqq C\left\|\hat{\phi}_{+}\right\|_{\infty}\left(\left\|\hat{\phi}_{+}\right\|_{\infty}+\left\|x \hat{\phi}_{+}\right\|_{\infty}\right)\left\|u_{1}-u_{2}\right\|_{Y}
$$

for any $u_{1}, u_{2} \in Y_{R}^{\alpha}(T)$. By (2.23) and (2.24), if $\left\|\hat{\phi}_{+}\right\|_{\infty}+\left\|x \hat{\phi}_{+}\right\|_{\infty}$ is small enough, then $J$ maps from $Y_{R}^{\alpha}(T)$ into itself and is a contraction on $Y_{R}^{\alpha}(T)$. Therefore $J$ has a unique fixed point $u \in Y_{R}^{\alpha}(T)$.

In either case the solution $u$ of $J u=u$ also satisfies (1.1) in $H^{-1,0}$ for all $t \geqq T$ by the same method as in the proof of Theorem 1 and $u$ extends to all times by the well known method of the Cauchy problem for (1.1) in the energy space $H^{1,0}$. We now prove $(1.14)_{+}$and $(1.15)_{+}$. In the same way as in $(2.14)_{+}$, we obtain

$$
\begin{aligned}
&\left\|\partial\left(u(t)-\exp \left(i S^{+}(t)\right) U(t) \phi_{+}\right)\right\|_{2} \\
& \leqq\left\|\partial\left(u(t)-\exp \left(i S^{+}(t)\right) U(t) M(-t) \phi_{+}\right)\right\|_{2}+\left\|\partial S^{+}(t) \cdot \exp \left(i S^{+}(t)\right) U(t)\left(\phi_{+}-M(-t) \phi_{+}\right)\right\|_{2} \\
&+\left\|\exp \left(i S^{+}(t)\right) U(t) \partial M(-t) \cdot \phi_{+}\right\|_{2}+\| \exp \left(i S^{+}(t) U(t)\left(\partial \phi_{+}-M(-t) \partial \phi_{+}\right) \|_{2}\right. \\
& \leqq R t^{-\alpha}+C\left(t^{-1} \log t\left\|\partial \hat{\phi}_{+}\right\|_{\infty}\left\|\hat{\phi}_{+}\right\|_{\infty}+t^{-(p-1) / 2}\left\|\partial \hat{\phi}_{+}\right\|_{\infty}\left\|\hat{\phi}_{+}\right\|_{\infty}^{p-2}\right) \\
& \cdot\left\|\phi_{+}-M(-t) \phi_{+}\right\|_{2}+\left\|\partial M(-t) \cdot \phi_{+}\right\|_{2}+\left\|\partial \phi_{+}-M(-t) \partial \phi_{+}\right\|_{2} \\
& \leqq R t^{-\alpha}+C\left(t^{-1} \log t\left\|\partial \hat{\phi}_{+}\right\|_{\infty}\left\|\hat{\phi}_{+}\right\|_{\infty}+t^{-(p-1) / 2}\left\|\partial \hat{\phi}_{+}\right\|_{\infty}\left\|\hat{\phi}_{+}\right\|_{\infty}^{p-2}\right) t^{-1}\left\|\phi_{+}\right\|_{0,2} \\
&+t^{-1}\left\|\phi_{+}\right\|_{0,1}+t^{-1}\left\|\phi_{+}\right\|_{1,2},
\end{aligned}
$$

which together with $(2.14)$ proves $(1.14)_{+}$. In the same way as in (2.15), we obtain for $0<\varepsilon<2(1-\alpha)$,

$$
\begin{aligned}
&\left\|\partial\left(u(t)-\exp \left(i S^{+}(t)\right) U(t) \phi_{+}\right)\right\|_{\infty} \\
& \leqq\left\|\partial\left(u(t)-\exp \left(i S^{+}(t)\right) U(t) M(-t) \phi_{+}\right)\right\|_{\infty}+\| \partial S^{+}(t) \\
& \cdot \exp \left(i S^{+}(t)\right) U(t)\left(\phi_{+}-M(-t) \phi_{+}\right) \|_{\infty} \\
&+\left\|\exp \left(i S^{+}(t)\right) U(t) \partial M(-t) \cdot \phi_{+}\right\|_{\infty}+\left\|\exp \left(i S^{+}(t)\right) U(t)\left(\partial \phi_{+}-M(-t) \partial \phi_{+}\right)\right\|_{\infty} \\
& \leqq\left\|\partial\left(u(t)-\exp \left(i S^{+}(t)\right) U(t) M(-t) \phi_{+}\right)\right\|_{\infty} \\
&+C(\varepsilon)\left(t^{-1} \log t\left\|\partial \hat{\phi}_{+}\right\|_{\infty}\left\|\hat{\phi}_{+}\right\|_{\infty}+t^{-(p-1) / 2}\left\|\partial \hat{\phi}_{+}\right\|_{\infty}\left\|\hat{\phi}_{+}\right\|_{\infty}^{p-2}\right) t^{-5 / 4+\varepsilon / 2}\left\|\phi_{+}\right\|_{0,2} \\
&+C t^{-3 / 2}\left\|\phi_{+}\right\|_{0,2}+C(\varepsilon) t^{-5 / 4+\varepsilon / 2}\left\|\phi_{+}\right\|_{1,2} .
\end{aligned}
$$


This and $(2.16)$ prove $(1.15)_{+}$. The required uniqueness follows in the same way as in the proof of Theorem 1. Q.E.D.

Proof of Corollary 2. Let $\phi_{+}$and $u$ be as in Theorem 2. In the same way as in the proof of $(1.10)_{+}$, we have

$$
\begin{aligned}
&\left\|\partial\left(u(t)-\exp \left(i S_{0}^{+}(t)\right) U(t) \phi_{+}\right)\right\|_{2} \\
& \leqq\left\|\partial\left(u(t)-\exp \left(i S^{+}(t)\right) U(t) \phi_{+}\right)\right\|_{2}+\left\|\left(\exp \left(i S^{+}(t)\right)-\exp \left(i S_{0}^{+}(t)\right)\right) U(t) \partial \phi_{+}\right\|_{2} \\
&+\left\|\left(\partial S^{+}(t)-\partial S_{0}^{+}(t)\right) U(t) \phi_{+}\right\|_{2}+\left\|\partial S_{0}^{+}(t)\left(\exp \left(i S^{+}(t)\right)-\exp \left(i S_{0}^{+}(t)\right)\right) U(t) \phi_{+}\right\|_{2} \\
& \leqq C t^{-\alpha}+C|\mu| t^{-(p-3) / 2}\left\|\hat{\phi}_{+}\right\|_{\infty}^{p-1}\left\|\partial \phi_{+}\right\|_{2}+C|\mu| t^{-(p-1) / 2}\left\|\partial \hat{\phi}_{+}\right\|_{\infty}\left\|\hat{\phi}_{+}\right\|_{\infty}^{p-2}\left\|\phi_{+}\right\|_{2} \\
&+C|\mu| t^{-(p-1) / 2} \log t\left\|\partial \hat{\phi}_{+}\right\|_{\infty}\left\|\hat{\phi}_{+}\right\|_{\infty}^{p}\left\|\phi_{+}\right\|_{2},
\end{aligned}
$$

which together with $(1.10)_{+}$proves $(1.18)_{+}$. In the same way as in the proof of $(1.13)_{+}$, we have

$$
\begin{aligned}
\|u(t)\|_{\infty} & \leqq\left\|u(t)-\exp \left(i S^{+}(t)\right) U(t) \phi_{+}\right\|_{\infty}+\left\|\exp \left(i S^{+}(t)\right) U(t) \phi_{+}\right\|_{\infty} \\
& \leqq C\left\|u(t)-\exp \left(i S^{+}(t)\right) U(t) \phi_{+}\right\|_{1,0}+\left\|\phi_{+}\right\|_{1} t^{-1 / 2} \\
& \leqq C t^{-\alpha}+\left\|\phi_{+}\right\|_{1} t^{-1 / 2} .
\end{aligned}
$$

This proves $(1.19)_{+}$. Q.E.D.

\section{References}

1. Barab, J. E.: Nonexistence of asymptotically free solutions for a nonlinear Schrödinger equation. J. Math. Phys. 25, 3270-3273 (1984)

2. Cazenave, T.: An introduction to nonlinear Schrödinger equations. Textos de Métodos Matemáticas vol. 22, Instituto de Matemática, Rio de Janeiro (1989)

3. Cazenave, T., Weissler, F. B.: The Cauchy problem for the critical nonlinear Schrödinger equation in $H^{s}$, Nonlinear Analysis. Theory Math. Appl. 14, 807-836 (1990)

4. Dong, G. C., Li, S. J.: On the initial value problem for a nonlinear Schrödinger equation. J. Differ. Eqs. 42, 353-365 (1981)

5. Flato, M., Simon, J., Taflin, E.: On global solutions of the Maxwell-Dirac equations. Commun. Math. Phys. 112, 21-49 (1987)

6. Friedman, A.: Partial Differential Equations, New York: Holt-Rinehart and Winston 1969

7. Ginibre, J.: Unpublished, untitled note

8. Ginibre, J., Velo, G.: On a class of non-linear Schrödinger equations III. Special theories in dimensions 1,2 and 3, Ann. Inst. Henri Poincaré, Physique théorique 28, 287-316 (1978)

9. Ginibre, J., Velo, G.: Scattering theory in the energy space for a class of nonlinear Schrödinger equations. J. Math. Pures Appl. 64, 363-401 (1985)

10. Glassey, R. T.: On the blowing-up solutions to the Cauchy problem for nonlinear Schrödinger equations. J. Math. Phys. 18, 1794-1797 (1977)

11. Hayashi, N., Nakamitsu, K., Tsutsumi, M.: On solutions of the initial value problem for the nonlinear Schrödinger equations in one space dimension. Math. Z. 192, 637-650 (1986)

12. Hayashi, N., Ozawa, T.: Scattering theory in the weighted $L^{2}\left(\mathbb{R}^{n}\right)$ spaces for some Schrödinger equations. Ann. Inst. Henri Poincaré, Physique théorique 48, 17-37 (1988)

13. Hayashi, N., Tsutsumi, M.: $L^{\infty}\left(\mathbb{R}^{n}\right)$-decay of classical solutions for nonlinear Schrödinger equations. Proceedings of the Royal Society of Edinburgh 104, 309-327 (1986)

14. Hayashi, N., Tsutsumi, Y.: Scattering theory for Hartree type equations. Ann. Inst. Henri Poincaré, Physique théorique 46, 187-213 (1987)

15. Kato, T.: On nonlinear Schrödinger equations. Ann. Inst. Henri Poincaré, Physique théorique 46, 113-129 (1987) 
16. Kato, T.: Nonlinear Schrödinger equations. In: Schrödinger Operators. Holden, H., Jensen, A. (eds.). Lecture Notes in Physics vol. 345. Berlin, Heidelberg, New York: Springer 1989

17. Makhankov, V. G.: Dynamics of classical solitons (in non-integrable systems). Phys. Reps. 35, 1-128 (1978)

18. Reed, M., Simon, B.: Methods of Modern Mathematical Physics, III, Scattering theory. New York: Academic Press 1979

19. Strauss, W. A.: Dispersion of low-energy waves for two conservative equations. Arch. Rat. Mech. Anal. 55, 86-92 (1974)

20. Strauss, W. A.: Nonlinear scattering theory at low energy. J. Funct. Anal. 41, 110-133(1981)

21. Thornhill, S. G., ter Haar, D.: Langmuir turbulence and modulational instability. Phys. Rep. 43, 43-99 (1978)

22. Tsutsumi, Y.: Scattering problem for nonlinear Schrödinger equations. Ann. Inst. Henri Poincaré, Physique théorique 43, 321-347 (1985)

23. Tsutsumi, Y.: Global Existence and Asymptotic Behavior of Solutions for Nonlinear Schrödinger Equations. Doctoral Thesis, University of Tokyo (1985)

24. Tsutsumi, Y.: $L^{2}$-solutions for nonlinear Schrödinger equations and nonlinear groups. Funkcialaj Ekvacioj 30, 115-125 (1987)

25. Tsutsumi, Y., Yajima, K.: The asymptotic behavior of nonlinear Schrödinger equations. Bull. Am. Math. Soc. 11, 186-188 (1984)

26. Weinstein, M. I.: Nonlinear Schrödinger equations and sharp interpolation estimates. Commun. Math. Phys. 87, 567-576 (1983)

27. Yajima, K.: Existence of solutions for Schrödinger evolution equations. Commun. Math. Phys. 110, 415-426 (1987)

28. Zakharov, V. E.: Collapse of Langmuir waves. Sov. Phys. JETP 35, 908-914 (1972)

29. Zakharov, V. E., Shabat, A. B.: Exact theory of two-dimensional self-focusing and one-dimensional self-modulation of waves in nonlinear media. Sov. Phys. JETP 37, 62-69 (1972)

30. Zakharov, V. E., Shabat, A. B.: Interaction between solitons in a stable medium. Sov. Phys. JETP 37, 823-828 (1973)

Communicated by J. Fröhlich 
